\title{
Simulation analysis of the diode laser spectral beam combining
}

\author{
Jing Li, Wenbin Qin*, Yinhua Cao, Yuntao Qiu, Youqiang Liu, Xiaodi Zeng, and Zhiyong \\ Wang \\ National Center of Laser Technology, Institute of Laser Engineering, Beijing University of \\ Technology, Beijing 100124, China
}

\begin{abstract}
For high power diode laser, the multi-wavelength combining is an effective technology of improving the beam brightness. Based on the combing technology, the diode laser bar is described, with the different sample grating in every emitter ridge, which can have stability of the wavelength and the single longitudinal mode. Mathematics computing model based on the spilt-step fourier (SSF) method combining is built using MatLab. It simulated the multi-wavelength combining process of emitters. In simulation, the diode laser beam successively go through a focus lens and a diffraction grating, respectively. In result, the model accords with the law of the combining which is verified by existing report. The simulation solution satisfied the reported experiment.
\end{abstract}

\section{Introduction}

With high conversion efficiency and minimization, diode laser is widely used in scientific research and industrial application. Extremely high brightness diode laser has become an increasing research hotspot and reports have been published about combining research to improve beam quality. Ghasemi SH [1] employs a stripe mirror plate and a V-Stack mirror and Gao X [2] uses two optical rectangular cubes and two stripe-mirror plates to shape diode laser beam to improve beam quality. However, the ways only change the shape of the diode laser and reduce the beam quality, while they could not meet the need of the better beam quality which is the same to that of a single emitter. For extremely high brightness output, spectral beam combining (SBC) has been proven to be an effective way. Several researches of SBC with diode laser bar or arrays have been reported in which the combining are gradually optimized to improve the output characteristic and power. Robin $\mathrm{K}$ Huang [3] demonstrated a fiber-coupled direct diode with a power level of $1040 \mathrm{~W}$ from a $200 \mu \mathrm{m}$ core diameter, 0.18 numerical aperture output fibers at single center wavelength. The fiber-coupled output corresponds to a Beam Parameter Product (BPP) of $18 \mathrm{~mm}$-mrad. To improve the efficiency of SBC and increase the number of combined laser, Jun Zhang [4] used a -1 st order fused silica transmission grating and combined a 970nm centimeter diode laser bar with 19 emitters, yielding a CW power of $50.8 \mathrm{~W}$, a holistic $\mathrm{M}^{2}$ of 10.9 , with $90.2 \%$ spectral combining efficiency. Zhanda Zhu [5] obtained the diode laser with $1.3 \times 11.6 \mathrm{M}^{2}$ in horizontal and vertical directions by spectral beam combing with a beam

${ }^{*}$ Corresponding author: wbqin@bjut.edu.cn 
shaping element inserted which rotated the beam of a $940 \mathrm{~nm}$ standard laser bar containing 19 laser elements by 90 degrees. From all of the above SBC reports, it can been seen that the beam combining is based on experiment. As far as the SBC is concerned, the numerical analysis model hasn't been reported yet and the analysis methods are only limited in processing experimental data.

In this letter, we analyze the beam transmit characteristics and build the combining model in spectral beam combining of laser bar based on the angular spectrum method. In section 2, we focus mainly on the split-step Fourier angular spectrum method. The efficiency of the split-step method depends on the frequency-domain resolution, with distribution of step sizes along the beam path of SBC. The SBC model is built according to the split-step Fourier (SSF) angular spectrum method. In section 3, according to the simulation result, we are able to get the SBC parameter of a 940nm diode laser bar, which consisted of 19 emitters. At the same time, the laser beam transmitting characteristics are displayed. The model could accurately analyze the transmit characteristics and simulate the SBC process taken less time.

\section{The split-step fourier (ssf) angular spectrum method}

Scalar diffraction theory [6] is applied to beam transmission of spectral beam combining. According to the source-plane optical field, the observation-plane field is given by Fresnel diffraction integral [7-8], as equation (1) show,

$$
U\left(\mathrm{x}_{2}, \mathrm{y}_{2}\right)=\frac{e^{i k \Delta z}}{i \lambda \Delta z} \int_{-\infty}^{\infty} U\left(\mathrm{x}_{1}, \mathrm{y}_{1}\right) e^{i \frac{k}{2 \Delta z}\left[\left(x_{1}-x_{2}\right)^{2}+\left(y_{1}-y_{2}\right)^{2}\right]} d x_{1} d y_{1}
$$

where $\Delta z$ is the transmit distance, $k$ is wave vector.

The right integral part could be regarded as the convolution, as equation (2) show,

$$
\int_{-\infty}^{\infty} U\left(\mathrm{x}_{1}, \mathrm{y}_{1}\right) e^{i \frac{k}{2 \Delta z}\left[\left(x_{1}-x_{2}\right)^{2}+\left(y_{1}-y_{2}\right)^{2}\right]} d x_{1} d y_{1}=U\left(\mathrm{x}_{1}, \mathrm{y}_{1}\right) \otimes e^{i \frac{k}{2 \Delta z}\left[\left(x_{1}\right)^{2}+\left(y_{1}\right)^{2}\right]}
$$

Assuming space spectrum of $U\left(\mathrm{x}_{1}, \mathrm{y}_{1}\right)$ space spectrum field is $u\left(f_{x 1}, f_{y 1}\right)$, we obtain equation (3)

$$
U\left(\mathrm{x}_{1}, \mathrm{y}_{1}\right)=f t^{-1}\left(u\left(f_{x 1}, f_{y 1}\right)\right)
$$

And equation (4)

$$
\frac{1}{i \lambda \Delta z} e^{-i \frac{k}{2 \Delta z}\left(x_{1}^{2}+y_{1}^{2}\right)}=f t^{-1}\left(e^{-i \pi \lambda \Delta z\left(f_{x 1}^{2}+f_{x 1}^{2}\right)}\right)
$$

Hence equation (5)

$$
U\left(\mathrm{x}_{2}, \mathrm{y}_{2}\right)=\int_{-\infty}^{\infty} u\left(f_{x 1}, f_{y 1}\right) e^{i k \Delta z-i \pi \lambda \Delta z\left(f_{x 1}^{2}+f_{x 1}^{2}\right)} e^{i\left(2 \pi f_{x 1} x_{1}+2 \pi f_{y 1} y_{1}\right)} d f_{x 1} d f_{y 1}
$$

where equation (6)

$$
e^{i k \Delta z-i \pi \lambda \Delta z\left(f_{x 1}{ }^{2}+f_{x 1}{ }^{2}\right)}=e^{i k \Delta z\left(1-\frac{1}{2}\left(\lambda^{2} f_{x 1}{ }^{2}+\lambda^{2} f_{y 1}{ }^{2}\right)\right)} \approx e^{i k \Delta z\left(1-\lambda^{2} f_{x 1}{ }^{2}-\lambda^{2} f_{y 1}{ }^{2}\right)^{1 / 2}}
$$

where $e^{i k \Delta z \sqrt{1-\lambda^{2} f_{x 1}^{2}-\lambda^{2} f_{y 1}^{2}}}$ is transfer function in angular spectrum method, as equation (7) show, 
$U\left(\mathrm{x}_{2}, \mathrm{y}_{2}\right)=f t^{-1}\left(u\left(f_{x 1}, f_{y 1}\right) e^{i k \Delta z \sqrt{1-\lambda^{2} f_{x 1}{ }^{2}-\lambda^{2} f_{y 1}{ }^{2}}}\right)=f t^{-1}\left\{f t\left(U\left(\mathrm{x}_{1}, \mathrm{y}_{1}\right)\right) e^{i k \Delta z \sqrt{1-\lambda^{2} f_{x 1}{ }^{2}-\lambda^{2} f_{y 1}{ }^{2}}}\right\}$

In split-step Fourier angular spectrum method [8], $\Delta z$ is the step size of beam transmission and the observation-plane field is the reverse Fourier transforms of the product of both the Fourier transforms of the source optical field and transfer function.

Figure 1 shows a schematic diagram of combining system containing a focus lens and transmission grating. The schematic structure contain a diode laser bar, FAC, SAC, focus lens and the grating. Every emitter beam of diode laser bar is collimated by FAC and SAC and combined through the focus lens and grating. In simulation, the change of beam field is considered on the field phase, while the intensity reduction is ignored with the high transmittance of lens. The phase of grating is expressed in both transmission grating and reflection grating.

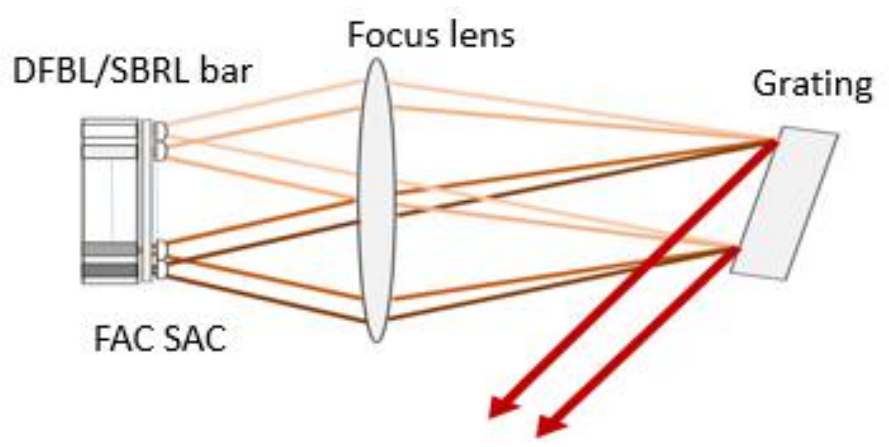

Fig. 1. Schematic diagram of combining system containing a focus lens and transmission grating.

In the model, beams of emitters with different wavelength, which are selected by algorithm in order to be similar to the external cavity, are combined. The beam combing is performed on the slow axis and the optical field is simplified to one dimension.

With the optical field simplified to, the Fresnel diffraction integral (1) is expressed as equation (8)

$$
U\left(\mathrm{x}_{2}\right)=\frac{e^{i k \Delta z}}{i \lambda \Delta z} \int_{-\infty}^{\infty} U\left(\mathrm{x}_{1}\right) e^{i \frac{k}{2 \Delta z}\left[\left(x_{1}-x_{2}\right)^{2}\right]} d x_{1}
$$

And equation (9)

$$
U\left(\mathrm{x}_{2}\right)=f t^{-1}\left(u\left(f_{x 1}\right) e^{i k \Delta z \sqrt{1-\lambda^{2} f_{x 1}^{2}}}\right)=f t^{-1}\left\{f t\left(U\left(\mathrm{x}_{1}\right)\right) e^{i k \Delta z \sqrt{1-\lambda^{2} f_{x 1}^{2}}}\right\}
$$

In order to improve the computing speed in simulation, Fast Fourier Transforms is applied and the obverse-plane field is satisfied, as equation (10) show,

$$
U\left(\mathrm{x}_{2}\right)=f f t^{-1}\left(u\left(f_{x 1}\right) e^{i k \Delta z \sqrt{1-\lambda^{2} f_{x 1}{ }^{2}}}\right)=f f t^{-1}\left\{f f t\left(U\left(\mathrm{x}_{1}\right)\right) e^{i k \Delta \sqrt{1-\lambda^{2} f_{x 1}{ }^{2}}}\right\}
$$

In the paper, we focus on the split-step fourier method [9]. In simulation, the path of beam is treated as the integral of split-step small beam path; Every small beam transmission is simulated applying on expression.

\section{Simulation result}


Two hypotheses are present. First one is that diode laser emitter field is fundamental mode field, and the other is that diode laser emitters with different wavelength work on the same emitting efficiency. In simulation, the diode laser bar with $940 \mathrm{~nm}$ central wavelength, 19 emitters which is collimated through FAC lens and SAC lens is combined through the lens $(\mathrm{f}=200 \mathrm{~mm})$ and the grating $(\mathrm{g}=600 / \mathrm{mm})$. The parameters in simulation are shown in the table 1 .

Table 1. the parameters in simulation.

\begin{tabular}{|l|c|}
\hline parameters & value \\
\hline Central wavelength (nm) & 940 \\
\hline Emitter number & 19 \\
\hline Gap of emitter (m) & $5 \mathrm{e}-4$ \\
\hline Zone of observe (m) & $4 \mathrm{e}-2$ \\
\hline Sampling rate (m) & $1 \mathrm{e}-7$ \\
\hline Split-step length (m) & $5 \mathrm{e}-3$ \\
\hline Focal length of focus lens (mm) & 200 \\
\hline Grating Grooves (/mm) & 600 \\
\hline Grating Littrow angle $\left(^{\circ}\right)$ & 17.45 \\
\hline
\end{tabular}

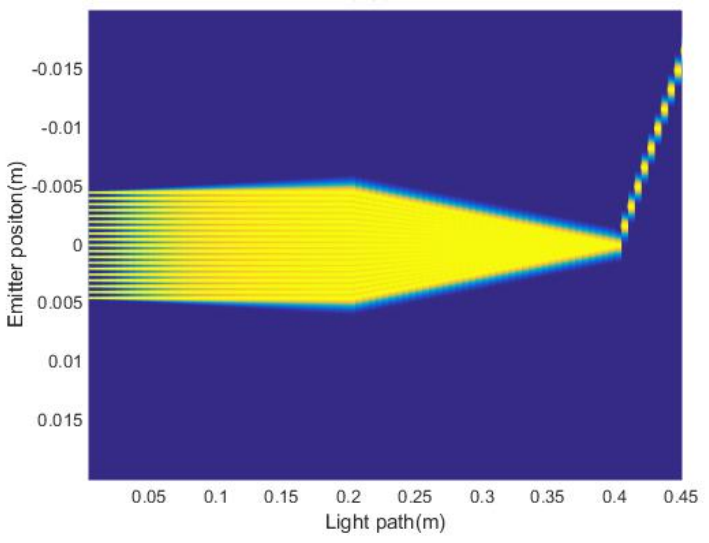

Figure 2 illuminates the combining process of the diode beam. Diode laser bar with 19 emitters is collimated by FAC and SAC and transmit $200 \mathrm{~mm}$ to the focus lens. After transmitting through the focus lens, the beam is focused on the grating which is placed on the focus point, which triangle groove is arrange in the slow axis of diode laser and where is a littrow angle between the laser beam and the grating normal.

Figure 3 shows the different position of emitters versus the emitter wavelength. The emitter beams is combining into a beam, with the wavelengths selected in the algorithm. The pitch of emitters is $500 \mu \mathrm{m}$; The line-symbol graph is drawn by real value, which fits into the red line with the slope -0.00306 , meaning the wavelength interval is $3.06 \mathrm{~nm}$. The data shown on the y-axis of graph is the wavelength of the symbol.

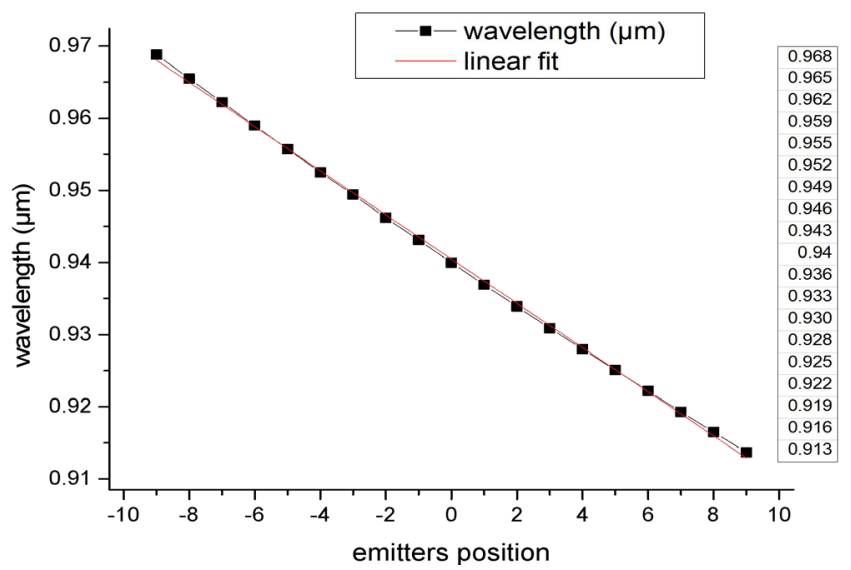

Fig. 3. Different position of emitters versus the emitter wavelength.

In figure 4 , the left figure is the light intensity of 19 emitters before combination. The 
different color expresses different wavelength and the emitters are laid with equal interval. The right figure demonstrates the combined beam with spectral beam. The beam through the grating is combined perfectly in the observation-plane field. There are two different values of intensity at the left and right of main combined beam, respectively. The two intensity only lose little energy and are ignored relative to main combined beam's intensity.
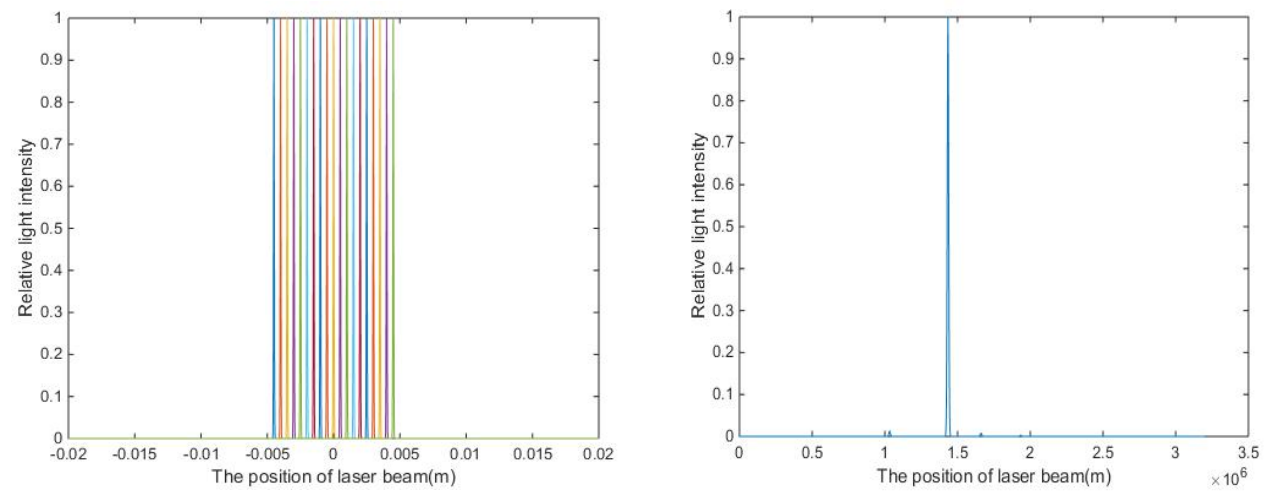

Fig. 4. Diode laser beam before and after combining. (Fig. 4(a) is relative light intensity of 19 emitter beams and fig. 4(b) is the combined beam.)

In order to review combining situation in detail, observing region is narrowed down to the zone in which the combined beam exists. Thus the combined laser beams is expanded, as shown in figure 5. It is 19 color that is overlapped together, meaning there is a combined beam of 19 beams overlapped together.

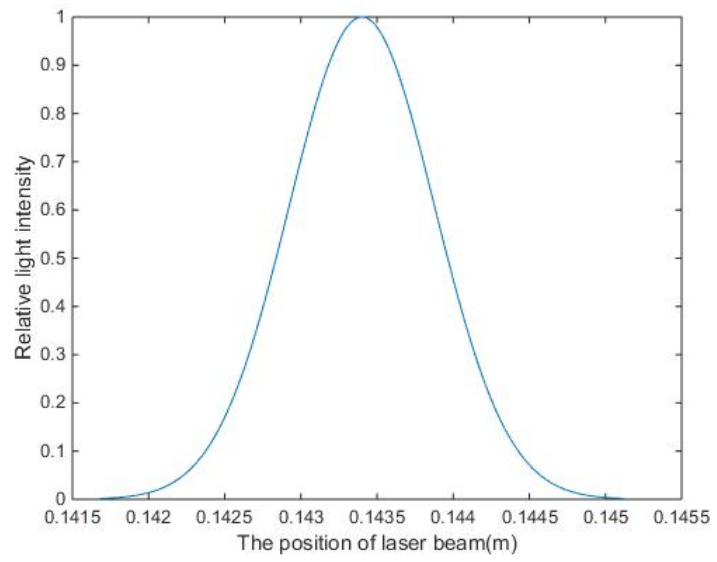

Fig. 5. Diode laser beam after combining.

The reason for this occurrence is that we neglect that diode laser emitters without $940 \mathrm{~nm}$ wavelength are not working on the highest emitting efficiency in simulation. All emitter intensity are expressed by the same function diagram and thus the 19 emitter beams is combined to a diagram.

\section{Conclusion}

We perfectly simulated the process of spectral beam combination of a $940 \mathrm{~nm}$ diode bar, which consists of 19 emitters, given the two hypotheses. 19 emitter beams with the same interval of $500 \mu \mathrm{m}$ are combined by spectral beam combine composed of the focus lens with 
the $200 \mathrm{~mm}$ focus length and the grating with $\mathrm{g}=600 / \mathrm{mm}$. The combined beam shares the same characteristics with the single emitter of diode bar.

The simulation presents a way based on split-step Fourier angular spectrum method which quickly simulate the spectral beam combine. It provides a guidance for the diode laser spectral beam combine for different parameter of lens and grating, as well as the idea of the different wavelength multi-single emitters combining.

\section{References}

1. S. H. Ghasemi, M.-R. Hantehzadeh, J. Sabbaghzadeh, D. Dorranian, M. Lafooti, V. Vatani, R. Rezaei-Nasirabad, A. Hemmati, A. A. Amidian, and S. A. Alavian, Beam shaping design for coupling high power diode laser stack to fiber., Appl. Opt., vol 50, no 18, pp 2927-30 (2011)

2. X. Gao, H. Ohashi, H. Okamoto, M. Takasaka, and K. Shinoda, Beam-shaping technique for improving the beam quality of a high-power laser-diode stack., Opt. Lett., vol 31, no 11, pp 1654-56 (2006)

3. R. K. Huang, B. Chann, J. D. Glenn, and B. B. Rd, Extremely high brightness , $\mathrm{kW}$ class fiber coupled diode lasers with wavelength stabilization, vol 8039, pp 1-10 (2011)

4. J. Zhang, H. Peng, X. Fu, Y. Liu, L. Qin, G. Miao, and L. Wang, CW 50W/M2 = 10.9 diode laser source by spectral beam combining based on a transmission grating, vol 21, no 3, pp 3627-32 (2013)

5. Z. Zhu, L. Gou, M. Jiang, Y. Hui, H. Lei, and Q. Li, High beam quality in two directions and high efficiency output of a diode laser array by, vol 22, no 15, pp 17804-809 (2014)

6. S. Thakur and S. N. Rao, Numerical Simulation of Optical Wave Propagation With examples in MATLAB, no 1 (2014)

7. M. Born and E. Wolf, Principles of optics: electromagnetic theory of propagation, interference ..., vol 1 (1999)

8. Joseph W. Goodman, Introduction to Fourier Optics (2005)

9. J. Sánchez-Curto and P. Chamorro-Posada, On a faster parallel implementation of the split-step Fourier method, Parallel Comput., vol 34, no 9, pp 539-49 (2008) 